\title{
HKSD for deformation measurement of nonrigid biological tissues from MR images
}

\author{
Jia Chen ${ }^{1, b}$, Ru-Han $\mathrm{He}^{1, \uparrow, b}$, Xu-Bing Zhang ${ }^{2, \mathrm{c}}$ and Jun Bai ${ }^{1, \mathrm{~d}}$ \\ ${ }^{1}$ School of Mathematics and Computer Science, \\ Wuhan Textile University, WuHan, China \\ ${ }^{2}$ Department of Regional Planning and Information Technology, \\ School of Public Administration. \\ China University of Geosciences, Wuhan, China

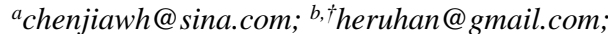 \\ cxubingnational@gmail.com; ${ }^{d} 1039122767 @ q q . c o m$
}

\begin{abstract}
Due to the nonlinear deformation of nonrigid and nonuniform tissues, it is challenging to accurately measure the displacements of feature points distributed on the inner parts, boundaries, and separatrices of tissue layers. To address this challenge, we propose a feature point matching technique called HKSD to measure MR 2-D slice deformation of nonuniform and nonrigid biological tissues. Finally, we describe patches based on the Heat Kernel Signature (HKS). We apply Gaussian function as weigh to noise 2D image. The experimental results showed that the proposed HKSD method outperformed the single SURF and SIFT methods.
\end{abstract}

Keywords: Feature point; Matching; Nonrigid; Image

\section{Introduction}

It is a wisdom to extract and match feature points of magnetic resonance (MR) images in Euclidean spaces [1-5]. In this paper, we propose a feature points matching technique (HKSD) using Riemannian spaces; we apply our technique to measure MR 2-D slice deformation of nonuniform and nonrigid biological tissues.

Recently, there has some new ways to interpret and analyze image $[6,7,8$, $9,10]$, such as Riemannian manifolds. These ways are non-Euclidean geometry. Also, heat diffusion theory has shown effectiveness in 3D shape analysis and recognition. In [11], they used heat diffusion theory to describe local features of deforming and scaling surfaces, and introduced a novel feature point descriptor named DaLI to non-rigid image transformations and illumination changes. 
Drawing inspiration from the DaLI [11], we propose a feature points matching technique (HKSD) using Riemannian spaces; we apply our technique to measure MR 2-D slice deformation of nonuniform and nonrigid biological tissues. We show how to embed 2D surfaces into 3D space, and how to describe patches based on the Heat Kernel Signature (HKS). Meanwhile, to increase robustness against 2D and intensity noise, we use multiple such descriptors in the neighborhood of a point, and weigh them by Gaussian function.

\section{Related Works}

Extraction and matching of the feature points, which should be robust against the change in illumination, scaling, rotation, and noise or slight distortion, is one of the most important methods used to detect the correspondences between the images. In the literature [12-24], a wide variety of feature point detectors and descriptors have already been proposed. Harris [14] proposed famous corner detector, which is based on the eigenvalues of the second-moment matrix, and this detector is not scale-invariant. Another famous detector is Scale Invariant Feature Transform (SIFT) approach, proposed by Lowe [12], [15], [16]. This approach approximated the Laplacian of Gaussian (LoG) by a Difference of Gaussians (DoG) filter. Florack [18] proposed moment invariants, but in [17], author pointed out SIFT outperforms other feature descriptors. Actually, There have some other well-known methods such as PCA-SIFT (Principle Component Analysis-SIFT) [13] and the GLOH (Gradient location-orientation histogram)[16], these methods are various refinements on the SIFT scheme. In [19], SURF (Speeded-UP Robust Feature) proposed by Bay, this scheme is more repeatable, distinctive, and robust than SIFT. However, SIFT and SURF is not invariant to general deformations [13], [21].

In [22], a nonrigid point matching algorithm RPM (robust point matching) propsed by Chui, this method can estimate the correspondence and nonrigid transformations between the two point-sets at the same time. Ling and Jacobs proposed GIH (Geodesic-Intensity Histogram) [23], which is a geodesic distance based deformation descriptor. However, this method assumed the deformation along different directions is isotropic. In [24], Tian used Hilbert scanning to reduce complexity. However, this scheme would be disabled when a point is located in the subdivision line of Hilbert scanning. 


\section{Methods}

\section{Feature point detection}

First, we need extract a large number of feature points in the MR images welldistributed. In this study, we adopt the Difference of Gaussian (DoG) operator to extract a number of feature points from a given deformed tissue and original tissue images respectively.

In what follows, we describe how to apply the DoG operator to generate a set of image features in three steps.

Firstly: We need identify potential interest points. We use a difference-ofGaussian function.

Secondly: Through calculate the Laplacian value of each key point found above, eliminating some low contrast points.

Finally: we use orientation to represent a key point, as a descriptor. We compute gradient magnitude and orientation to select Gaussian smoothed image, and form an orientation histogram. According the histogram, assign orientation to the key points.

Now, we need embed a image patch in a $3 \mathrm{D}$ space. A point of interest $\mathrm{p}$ is a center in a $2 \mathrm{D}$ image patch $\mathrm{S}$. Based on the diffusion geometry theory, mapping of the patch $\mathrm{S}$ to a $3 \mathrm{D}$ Riemannian manifold $\mathrm{M}(\mathrm{f}: \mathrm{S} \rightarrow \mathrm{P}$ ). We use follow function:

$$
\mathrm{f}: \mathrm{x} \rightarrow(\mathrm{x}, \mathrm{y}, \alpha \mathrm{S}(\mathrm{x})) \forall \mathrm{x} \in \mathrm{P}
$$

where $\mathrm{S}(\mathrm{x})$ is the pixel intensity at $x=(x, y)^{T}$, and $\alpha$ is a parameter which controls the amount of gradient magnitude preserved in the descriptor.

\section{Deformation invariant descriptor}

Based on heat diffusion equation, we can character each patch S:

$$
\left(\Delta N+\frac{\partial}{\partial t}\right) \mathrm{v}(x, t)=0,
$$

Where $\Delta N$ is the Laplace-Beltrami operator. We called $\mathrm{L}(\mathrm{x}, \mathrm{y}, \mathrm{t})$ which is a heat equation heat kernel. We use this equation represent the amount of heat between two points at some time t. We also use follow equation to express heat kernel for a compact manifold M [25,26]:

$$
L(x, y, t)=\sum_{i=0}^{\infty} \mathrm{g}^{-\lambda_{i} t} \ell_{i}(x) \ell_{i}(y),
$$


Where $\left\{\lambda_{i}\right\}$ and $\left\{\ell_{i}\right\}$ are the eigenvalues and eigenvectors of $\Delta N$. Actually, this equation (3) is equation (2)'s expansion. So, according to the equation (3), we describe a point $\mathbf{s}$ on $M$ using the Heat Kernel Signature:

$$
H K S(\mathrm{~s}, t)=L(s, s, t)=\sum_{i=0}^{\infty} g^{-\lambda_{i} t} \ell_{i}^{2}(s),
$$

It is isometrically-invariant, and adequate for capturing both the local properties of the shape around $\mathbf{s}$ (when $t \rightarrow 0$ ) and the global structure of $M$ (when $t \rightarrow \infty$ ).

But, the heat kernel may be unstable along the spatial domain, because when the shapes are irregular, the HKS of neighboring points are very similar. So, this HKS is sensitive to some 2D coordinates of keypoints. In this paper, we introduce a Gaussian function, this function is the distance to the center of the patch. Thus, we define the following Deformation Invariant descriptor:

$$
S P(\mathrm{~s}, t)=[H K S(x, t) \cdot G(x ; \mathrm{s}, \delta)]_{\forall x \in S},
$$

where $G(\mathbf{x} ; \mathbf{S}, \delta)$ is a $2 \mathrm{D}$ Gaussian function, $\mathbf{S}$ is mean and $\delta$ is standard deviation.

\section{Handling in-plane rotation and scaling}

Actually, SP descriptor is not designed for rotation and scaling. We designed Gaussian function is for the 2D noise, so we need all the pixels in the patch and their spatial relations.

In order to handle this situation, during the matching process we will consider several rotated and scaled copies of the descriptors. Therefore, given $\mathrm{SP}(\mathrm{s} 1)$ and SP(s2) we will compare them based on the following metric:

$$
\begin{gathered}
d\left(\mathrm{~s}_{1}, \mathrm{~s}_{2}\right)=\arg \min \left\|T_{\theta_{i, s_{j}}}\left(S P\left(\mathrm{~s}_{1}\right)\right)-S P\left(\mathrm{~s}_{2}\right)\right\| \\
\theta_{i}, s_{j}
\end{gathered}
$$

where $\|\cdot\|$ denotes the $\mathrm{L}_{2}$ norm and $T_{\theta_{i}, s_{j}}(S P(\mathrm{~s}))$ rotates and scales SP(s) by an angle $\theta_{\mathrm{i}}$ and a scale parameter $\mathrm{s}_{\mathrm{j}}$, respectively. These parameters are chosen among a discrete set of values $\theta$ and $\mathrm{s}$.

\section{Experiments}

We compared the methods of SIFT, SURF, and HKSD experimentally. The initial and deformed MR images of volunteers' calves are shown in Figure 1. 
The images are taken by the $0.5 \mathrm{~T}$ open MRI device, of which the FOV is $24 \times 24 \mathrm{~cm}$. The distance between two adjacent slices is $2 \mathrm{~mm}$. For the SIFT and SURF methods, each of the image pyramids consisted of 3 octaves, with every octave having 4 levels with different scales. After the feature points were extracted, the NN/SCN (the ratio of the nearest and second nearest neighbors) of SURF distance was adopted to match the feature points of the initial and the deformed MR images.

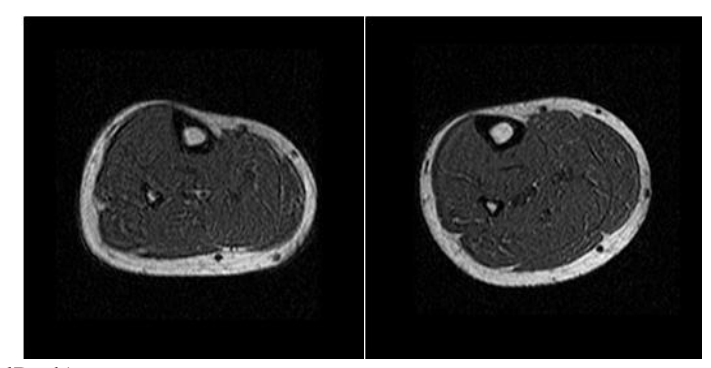

Figure 1. Calf push (CalPush)

Table 1 show that compared with SIFT and SURF, HKSD significantly improves the number of correctly matched pairs. In this example, HKSD increases the correctly matched pairs of SIFT and SURF by $21 \%$ and $20 \%$, respectively. The results confirm that our HKSD is capable of offering accurate deformation measurements of no rigid deformed tissues.

Table 1. comparison of Experimental Results of Four Methods

\begin{tabular}{|l|l|l|l|l|l|}
\hline MR image & Method & NM & NCM & NWM & Ratio(\%) \\
\hline \multirow{4}{*}{ CalPush } & SIFT & 63 & 18 & 45 & 28.57 \\
\cline { 2 - 6 } & SURF & 66 & 14 & 52 & 21.21 \\
\cline { 2 - 6 } & HKSD & 63 & 47 & 16 & 74.95 \\
\hline
\end{tabular}

NM: Number of the matched pairs; NCM: Number of correctly matched pairs; NWM Number of incorrectly matched pairs; Ratio: matching accuracy.

\section{Conclusion}

In this study, we proposed a feature point matching scheme called HKSD to offer MR 2-D slice deformation measurements. We carried out three phases in the development of HKSD. First, we used DoG detector and SIFT descriptor to extract the feature points. Then, embed 2D surfaces into 3D space. Finally, we embedded Riemannian manifolds into the familiar HKSD space and use HKS described the spatch.

we propose a feature points matching technique using Riemannian spaces; we apply our technique to measure MR 2-D slice deformation of nonuniform 
and nonrigid biological tissues. We show how to embed 2D surfaces into 3D space, and how to describe patches based on the Heat Kernel Signature (HKS). Meanwhile, to increase robustness against 2D and intensity noise, we use multiple such descriptors in the neighborhood of a point, and weigh them by Gaussian function. The experimental results showed that the proposed HKSD method outperformed the single SURF and SIFT methods.

\section{Acknowledgment}

This research was supported by the Education Bureau of Hubei Province under Grants Q20151602.

\section{References}

[1] Yimin Hou, Nini Rao, Xiangmin Lun, and Feng Liu, " Gait Object Extraction and Recognition in Dynamic and Complex Scene Using Pulse Coupled Neural Network and Feature Fusion," Journal of Medical Imaging and Health Informatics. 4(2), 2014, pp. 325-330.

[2] P. L. Zhang, S. Hirai, and K. Endo, K., A feature matching-based approach to deformation fields measurement from MR images of non-rigid object, International Journal of Innovative Computing, Information and Control. 4, 1607-1625(2008).

[3] Jia Chen, Xubing Zhang, Yuanquan Xu, and Ruhan He, "SIFT and Preserving Topology Structures of Local Neighborhood: Matching Feature Point in Deformation Measurement of Nonrigid Biological Tissues from Magnetic Resonance Images", J. Med. Imaging Health Inf. 5, 477-485 (2015).

[4] K. Selva Bhuvaneswari and P. Geetha. Semantic Segmentation and Categorization of Brain MRI Images for Glioma Grading. Journal of Medical Imaging and Health Informatics. 4(4), 554-566 (2014).

[5] Z. K. Wang, K. Namima, and S. Hirai, Physical parameter identification of rheological objects based on measurements of deformation and force, in Conf. 2009 IEEE Int. Conf. Robotics and Automation (ICRA 2009),( 2009) 1047-1052; Kobe, Japan.

[6] M. T. Harandi, C. Sanderson, S. Shirazi, and B. C. Lovell. Graph embedding discriminant analysis on Grassmannian manifolds for improved image set matching. In IEEE Conf. Computer Vision and Pattern Recognition (CVPR), pages 2705-2712, 2011.

[7] Y. M. Lui. Advances in matrix manifolds for computer vision. Image and Vision Computing, (in press). doi: 10.1016/j.imavis.2011.08.002. 
[8] X. Pennec. Intrinsic statistics on Riemannian manifolds: Basic tools for geometric measurements. Journal of Mathematical Imaging and Vision, 25(1):127-154, 2006.

[9] R. Subbarao and P. Meer. Nonlinear mean shift over Riemannian manifolds. Int. J. Computer Vision, 84(1):1-20, 2009.

[10] P. Turaga, A. Veeraraghavan, A. Srivastava, and R. Chellappa. Statistical computations on grassmann and stiefel manifolds for image and videobased recognition. IEEE Trans. Pattern Analysis and Machine Intelligence, 33(11):2273-2286, 2011.

[11] F. Moreno-Noguer. Deformation and illumination invariant feature point descriptor. In IEEE Conference on Computer Vision and Pattern Recognition, pages 1593-1600, 2011.

[12] D. Lowe, Distinctive image features from scale-invariant keypoints, International Journal of Computer Vision. 60, 91-110(2004).

[13] Y. Ke, and R. Sukthankar, PCA-SIFT: A more distinctive representation for local image descriptors, Computer Vision and Pattern Recognition. 2, 506-513( 2004).

[14] C. Harris, and M. Stephens, A combined corner and edge detector, In Proc. Proceedings of the 4th Alvey Vision Conference, (1988) 147-151; Manchester, UK.

[15] K. Mikolajczyk, and C. Schmid, Indexing based on scale invariant interest points, In Proc. Proceedings of the 8th International Conference on Computer Vision, (2001) 525-531; Vancouver, Canada.

[16] K. Mikolajczyk, and C. Schmid, Scale and affine invariant interest point detectors. International Journal of Computer Vision. 60, 63-86( 2004).

[17] K. Mikolajczyk, and C. Schmid, A performance evaluation of local descriptors. IEEE Transactions on Pattern Analysis and Machine Intelligence. 27, 1615-1630(2005).

[18] L. M. J. Florack, Haar, B. M. t. Romeny, J. J. Koenderink, and M. A. Viergever, General intensity transformations and differential invariants, Journal of Mathematical Imaging and Vision. 4, 171-187(1994).

[19] F. Mindru, T. Tuytelaars, Gool, L.Van, and T. Moons, Moment invariants for recognition under changing viewpoint and illumination, Computer Vision and Image Understanding. 94, 3-27(2004).

[20] H. Bay, T. Tuytelaars, and L. V. Gool, SURF: Speeded Up Robust Features, 9th European Conference on Computer Vision, (2006) 404 -417; Graz, Austria.

[21] H. Bay, A. Ess, T. Tuytelaars, and L. V. Gool, Speeded-up Robust Features (SURF), Computer Vision and Image Understanding. 110, 346-359(2008). 
[22] H. Chui and A. Rangarajan, A new algorithm for non-rigid point matching, in Conf. IEEE conference on Computer Vision and Pattern Recognition, (2000) 44-51; Hilton Head Island, SC, USA.

[23] H. Lin and D. W. Jacobs. Deformation invariant image matching. In Proc. ICCV, (2005) 1466-1473; Beijing, China.

[24] H. Cheng, Z. C. Liu, N. Zheng and J. Yang. A Deformable Local Image Descriptor, In Proc, CVPR, (2008) 1-8; Anchorage, AK. 